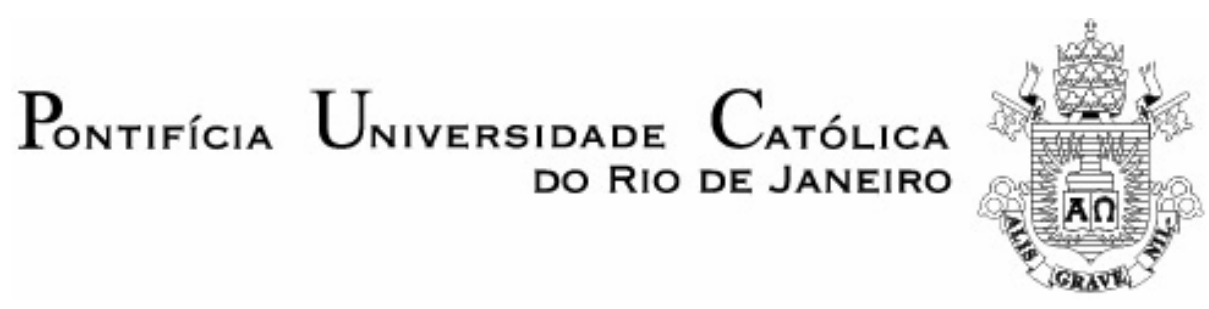

Gabriela Alejandra Huamán Pino

Biossorção de Metais Pesados Utilizando Pó da Casca de Coco Verde (Cocos nucifera)

\begin{abstract}
Dissertação de Mestrado
Dissertação apresentada como requisito parcial para obtenção do título de Mestre pelo Programa de PósGraduação em Engenharia Metalúrgica do Departamento de Ciência dos Materiais e Metalurgia da PUC-Rio.
\end{abstract}

Orientadora : Luciana Maria Souza de Mesquita Co-orientador: Mauricio Leonardo Torem 


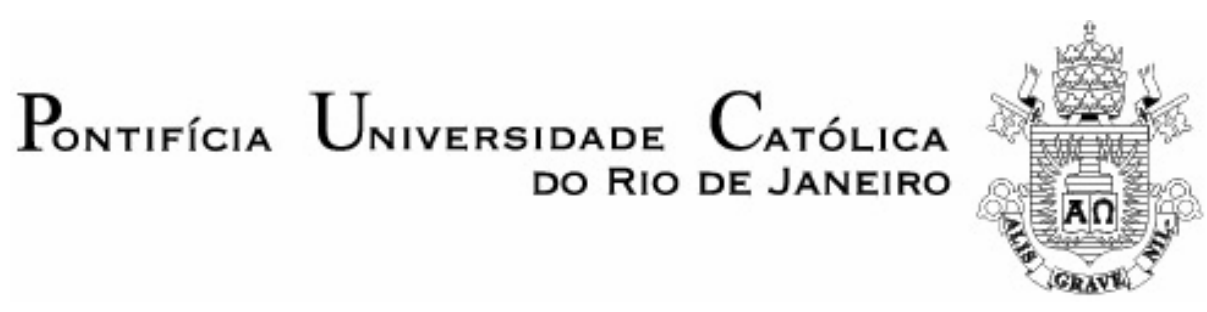

Gabriela Alejandra Huamán Pino

\section{Biossorção de Metais Pesados Utilizando Pó da Casca de \\ Coco Verde (Cocos nucifera)}

Dissertação apresentada como requisito parcial para obtenção do grau de Mestre em Engenharia Metalúrgica e de Materiais pelo Programa de Pós-Graduação em Engenharia Metalúrgica do Departamento de Ciência dos Materiais e Metalurgia da PUC-Rio. Aprovada pela Comissão Examinadora abaixo assinada.

Prof. Luciana Maria Souza de Mesquita

Orientadora

Departamento de Ciência dos Materiais e Metalurgia - PUC-Rio

Prof. Mauricio Leonardo Torem

Co-orientador

Departamento de Ciência dos Materiais e Metalurgia - PUC-Rio

Dr. Gustavo Adolfo Saavedra Pinto

Empresa Brasileira de Pesquisa Agropecuária - Embrapa

Prof. Selma Gomes Ferreira Leite Escola de Química - UFRJ

Prof. Roberto José de Carvalho

Departamento de Ciência dos Materiais e Metalurgia - PUC-Rio

Prof. José Eugenio Leal

Coordenador Setorial de pos-Graduação do Centro Técnico Científico - PUC-Rio

Rio de Janeiro, 30 de março de 2005 
Todos os direitos reservados. É proibida a reprodução total ou parcial do trabalho sem autorização da universidade, da autora e do orientador.

Gabriela Alejandra Huamán Pino

Graduou-se em Engenharia Química na UNSA (Universidad Nacional de San Agustín) em 2000 Arequipa Perú

Ficha Catalográfica

Huamán Pino, Gabriela Alejandra
Biossorção de Metais Pesados Utilizando Pó da
Casca de Coco Verde (cocos nucifera) / Gabriela
Alejandra Huamán Pino ; orientadora: Luciana Maria
Souza de Mesquita, co-orientador Mauricio Leonardo
Torem. - Rio de Janeiro : PUC-Rio, Departamento de
Ciência dos Materiais e Metalurgia, 2005.
113 f. ; $30 \mathrm{~cm}$
Dissertação (mestrado) - Pontifícia Universidade
Católica do Rio de Janeiro, Departamento de Ciência dos
Materiais e Metalurgia.
Inclui referências bibliográficas
1. Ciência dos Materiais e Metalurgia - Teses.
2. Biossorção. 3. Metais pesados. 4. Cocos nucifera. 5.
Biosorvente. 6. Efluentes. I. Mesquita, Luciana Maria
Souza de. II. Torem, Mauricio Leonardo III. Pontifícia
Universidade Católica do Rio de Janeiro. Departamento
de Ciência dos Materiais e Metalurgia. III. Título.


Para chegar, onde quer que seja, não é preciso dominar a força, mas sim, é preciso ter paciência, e é preciso, antes de tudo, sonhar. 
Este trabalho afastou-me ainda mais de casa, mas o amor e apoio dos meus pais, Antonio e Carmen, e dos meus irmãos, Antonio, Fernanda e Joaquín foi incondicional. As cartas, os telefonemas e os e-mails, trouxeram-me alegria, boas recordações e ânimo para continuar. Dedico este trabalho a vocês. 


\section{Agradecimentos}

A Deus, por ter me ajudado a chegar até aqui.

A Professora Luciana Maria Souza de Mesquita e ao Professor Mauricio Leonardo Torem, pelo estímulo, paciência, compreensão e por terem se mostrado grandes amigos nos momentos difíceis.

A Embrapa, na pessoa do doutor Gustavo Saavedra, pelo fornecimento da biomassa para a realização desta dissertação.

Ao CETEM pela delicadeza da cessão de seus laboratórios, a engenheira Marisa Bezerra de Mello Monte e a técnica Antonieta Middea pela disponibilização e treinamento no uso do equipamento de potencial zeta.

Ao departamento da química da PUC-Rio na pessoa do professor Reinaldo Calixto de Campos e ao técnico Rodrigo Gonçalves pela ajuda com as análises de absorção atômica.

A todos os professores pelos ensinamentos, e aos funcionários do departamento de ciência dos Materiais e Metalurgia, DCMM, ao técnico Marcos Bella Cruz Silva pelo apoio nas análises de absorção atômica, ao técnico Marcos Henrique de Pinho Mauricio pelas análises no MEV/EDS, a Lusinete pela paciência.

Aos meus amigos Ysrael, por ser "ele" quando eu precisei, a Mónica, o Omar, a Lesly e a Clety, pela companhia, conselhos e apoio dados ao longo do trabalho.

A os meus amigos "da montanha" por momentos inesquecíveis que fizeram esta caminhada mais leve e cuja companhia tornou a distância de casa menos sofrida.

A minha família, por tudo.

Ao CNPq pelo apoio financeiro dispensado durante o curso de mestrado. 


\section{Resumo}

Huamán Pino, Gabriela Alejandra. Biossorção de Metais Pesados Utilizando Pó da Casca de Coco Verde (Cocos nucífera). Rio de Janeiro, 2005. 113p. Dissertação de Mestrado - Departamento de Ciência dos Materiais e Metalurgia, Pontifícia Universidade Católica do Rio de Janeiro.

A poluição por metais pesados vem se tornando um sério problema ambiental. O uso de biomassas como material sorvente para a destoxificação de efluentes industria is contendo metais aparece como uma alternativa promissora às tecnologias existentes. O objetivo desta dissertação foi determinar a capacidade de adsorção da casca de coco (Cocos nucifera) para diferentes metais pesados. No processo de biossorção foram ava liados o pH da solução, a concentração inicial dos íons metálicos, o tamanho de partícula e a cinética do processo. As características da superfície da casca de coco foram investigadas utilizando medições de potencial zeta e análises com microscópio eletrônico de varredura. Obtiveram-se altas percentagens de remoção para o $\mathrm{Cd}, \operatorname{Cr}(\mathrm{III})$ e $\mathrm{Cr}(\mathrm{VI})$ dentre as seis espécies metálicas estudadas (As(V), Cd, $\mathrm{Cr}(\mathrm{III}), \mathrm{Cr}(\mathrm{VI}), \mathrm{Ni}$ e Zn). Os valores ótimos de $\mathrm{pH}$ utilizados foram de 7 para o cádmio e cromo (III) e de 2 para o cromo (VI). Em concentrações inicias inferiores a $100 \mathrm{mg} / \mathrm{L}$ foram obtidas percentagens de remoção superiores a 95\%, 85\% e 80\% para o cádmio, cromo (III) e cromo (VI), respectivamente. Em concentrações inicias menores que $1000 \mathrm{mg} / \mathrm{L}$, a percentagem de remoção para o cádmio e o cromo (III) foi de $80 \%$ e $85 \%$, respectivamente. Foram utilizados os modelos de Langmuir e Freundlich para avaliar a capacidade de adsorção de metais pesados pela casca de coco. O modelo de Langmuir mostrou-se mais adequado para o cádmio e o cromo (VI), enquanto que para o cromo (III) o modelo de Freundlich foi o mais adequado. A cinética do processo de biossorção foi estudada para o cádmio mediante as análises integral e diferencial. Foi observado que o processo de biossorção de cádmio por casca de coco verde obedece a um modelo de pseudo-segunda ordem. Os resultados apresentados mostram que a casca de coco apresenta as características apropriadas para o processo de biossorção de cádmio, cromo (III) e cromo (VI) e que pode ser utilizada como uma alternativa aos processos clássicos de destoxificação de efluentes.

\section{Palavras-chave}

biossorção; metais pesados; cocos nucifera; biossorvente; e fluentes. 


\section{Abstract}

Huamán Pino, Gabriela Alejandra. Biossorption of Heavy metals on green coconut powder. Rio de Janeiro, 2005. 113p. Master Dissertation Department of Materials Science and Metallurgical, Pontifical University Catholic of Rio de Janeiro.

Heavy metal pollution has become one of the most serious problems today, and the use of organic material biomass for the detoxification of industrial effluents for environmental protection offers a potential alternative to existing trea tment technologies. The aim of this work was determined the biosorption capacity of coconut fibers (Cocos nucifera) for different heavy metals. The effect of $\mathrm{pH}$, initial concentration, size of particle and kinetics were studied. The characteristics of the surface of coconut fibers had been investigated using measurements of potential zeta and analyses with scanning electron microscope. The removal was found to be higher for cadmium, chromium (III) e chromium (VI) among the 6 metallic species studied (As, Cd, Cr (III), Cr (VI), Ni e Zn). The values of $\mathrm{pH}$ used were 7 for cadmium and chromium (III) and of 2 for chromium (VI). For cadmium, chromium (III) and chromium (VI), with an initial metal concentration lower than $100 \mathrm{mg} / \mathrm{L}$, removal rates of $95 \%, 85 \%$ and $80 \%$ respectively had been observed. For cadmium and chromium (III), with an initial concentration lower than $1000 \mathrm{mg} / \mathrm{L}$, removal rates of $80 \%$ and $85 \%$ respectively were found. The adsorption capacity of coconut fibers for heavy metals had been evaluated using Langmuir and Freundlich adsorption isotherms. The Langmuir's model gave best results for cadmium and chromium (VI) and Freundlich's model for chromium (III). The kinetics of the process was studied just for cadmium, because it presented the best removal rates. The kinetics study showed that the biosorption process followed a pseudo second order model. This suggests that coconut fibers represent a good option for biosorption process and can be used as an alternative to the classical technologies for effluent decontamination.

\section{Keywords}

biosorption; heavy metals; cocos nucifera; biosorbent, wastewaters. 


\section{Sumário}

1 INTRODUÇÃO 16

$\begin{array}{ll}\text { 1.1. Objetivo } & 18\end{array}$

2 REVISÃO BIBLIOGRÁFICA 19

2.1. Metais Pesados 19

2.1.1. Arsênio 23

2.1.2. Cádmio 23

2.1.3. Cromo 24

2.1.4. Níquel 25

2.1.5. Zinco 26

2.2. Métodos Convencionais de Remoção de Metais Pesados 26

2.3. Biossorção 28

2.4. Biomassas 33

2.4.1. Exemplos de Biomassas Inativas Como Material Biossorvente 36

2.5. Cocos nucifera 39

2.6. Adsorção 44

2.6.1. Tipos de Adsorção 44

2.6.2. Isotermas de adsorção $\quad 45$

2.6.3. Isoterma de Langmuir $\quad 49$

2.6.4. Isoterma de Freundlich 51

2.7. Potencial zeta ( $(\xi) \quad 51$

2.8. Cinética de Remoção de Metais 53

2.8.1. Análise pelo Método Integral $\quad 54$

2.8.1.1. Teste para reação de ordem zero 56

2.8.1.2. Teste para reação de primeira ordem 56

2.8.1.3. Teste para reação de segunda ordem 57

2.8.1.4. Modelo de pseudo primeira ordem 58

2.8.1.5. Modelo de pseudo segunda ordem 59 
3 MATERIAIS E MÉTODOS 61

3.1. Material Biossorvente $\quad 61$

3.2. Soluções $\quad 62$

3.2.1. Solução de Arsênio 62

3.2.2. Solução de Cádmio 62

3.2.3. Solução de Cromo 63

3.2.4. Solução de Níquel 63

3.2.5. Solução de Zinco 63

3.2.6. Soluções para o ajuste do $\mathrm{pH} \quad 63$

3.3. Experimentos de Biossorção 63

3.3.1. Experimentos com Variação do pH 64

3.3.2. Experimentos com Variação do Tamanho de Partícula 64

3.3.3. Isotermas de Adsorção 64

3.4. Determinações Analíticas $\quad 64$

3.4.1. Determinação das Concentrações dos Metais por Espectrofotometria de Absorção Atômica $\quad 64$

3.4.2. Determinação do Potencial Zeta 65

3.5. Caracterização por MEV e EDS 66

3.6. Experimentos para Determinar a Cinética do Processo 67

4 RESULTADOS E DISCUSSÃO 68

4.1. Caracterização da Biomassa 68

4.1.1. Análise Granulométrica do Pó de Casca de Coco 68

4.1.2. Determinação do Potencial Zeta 68

4.1.3. Análises de MEV e EDS 70

4.2. Ensaios de Biossorção

4.2.1. Efeito do $\mathrm{pH} \quad 72$

4.2.2. Efeito do Tamanho de Partícula 78

4.2.3. MEV e EDS $\quad 80$

4.2.4. Variação da Concentração Inicial dos Metais 84

4.2.5. Isotermas de adsorção 86

4.3. Cinética de Biossorção de Cd 94

4.3.1. Análise pelo Método Integral 95

4.3.1.1. Teste para Ordem Zero 96

4.3.1.2. Teste para Primeira Ordem 96

$\begin{array}{ll}\text { 4.3.1.3. Teste para Segunda Ordem } & 97\end{array}$ 
4.3.1.4. Modelo de Pseudo Primeira Ordem 98

4.3.1.5. Modelo de Pseudo Segunda Ordem 99

5 CONCLUSÕES 102

6 SUGESTÕES PARA TRABALHOS FUTUROS 104

7 REFERÊNCIAS BIBLIOGRÁFICAS 105 


\section{Lista de figuras}

Figura 1-Resíduos produzidos nos países desenvolvidos. 20

Figura 2 - Diagrama esquemático de um processo de biossorção. 30

Figura 3 - Partes do coco verde. 41

Figura 4 - Espectro infravermelho do pó de casca de coco . 43

Figura 5-Formas comuns de uma isoterma de adsorção. 47

Figura 6-Classificação das isotermas. $\quad 48$

Figura 7- Modelo da dupla camada elétrica. $\quad 52$

Figura 8 - Fluxograma das etapas de obtenção do pó de casca de coco verde 61

Figura 9- Pó de casca de coco verde. 62

Figura 10 - Medição do potencial zeta do pó da casca de coco. 69

Figura 11- Microfotografias de partículas de pó ce casca de coco. 70

Figura 12 - EDS da partícula de casca de coco apresentada na Figura 11(a) 71

Figura 13- EDS da partícula de casca de coco apresentada na Figura 11(d) 71

Figura 14 - Capacidade de adsorção do pó de casca de coco em função do pH para o cádmio, cromo (III) e cromo (VI). 72

Figura 15- Diagrama de especiação do cromo (III). 73

Figura 16- Diagrama especiação do cádmio. $\quad 74$

Figura 17 - Capacidade de adsorção eb pó de casca de coco verde em função do pH para o arsênio, níquel e zinco . $\quad 75$

Figura 18- Diagrama de especiação do zinco. 76

Figura 19- Diagrama de especiação do arsênio (V). 77

Figura 20- Percentagem de remoção dos íons Cd, $\mathrm{Cr}$ (III), e Cr (VI). 79

Figura 21 - Microfotografias de partículas de casca de coco carregadas com íons $\mathrm{Cr}$ (III). $\quad 81$

Figura 22- EDS da partícula de casca de coco carregada com íons de Cr (III). 81

Figura 23 - Microfotografias de partículas de casca de coco carregadas com íons $\mathrm{Cd}$. 82

Figura 24 - EDS da partícula de casca de coco carregada com íons de Cd. 82

Figura 25 - Microfotografias de partículas de casca de coco carregadas com íons $\mathrm{Cr}(\mathrm{VI})$.

Figura 26 - EDS da partícula de casca de coco carregada com íons de $\mathrm{Cr}$ (VI). 83

Figura 27 - Percentagem de remoção de Cd em função da conc. inicial. $\quad 84$ 
Figura 28- Percentagem de remoção de $\mathrm{Cr}$ (III) em função da conc. inicial 85

Figura 29 - Percentagem de remoção de $\mathrm{Cr}(\mathrm{VI})$ em função da conc. inicial. $\quad 85$

Figura 30- Isoterma de adsorção para o Cd. 88

Figura 31- Isoterma de adsorção para o $\mathrm{Cr}$ (III). 88

Figura 32- Isoterma de adsorção para o $\mathrm{Cr}(\mathrm{VI})$. $\quad 89$

Figura 33 - Linearização da isoterma de Langmuir. 90

Figura 34- Linearização da isoterma de Freundlich. 90

Figura 35- Cinética da biossorção do cádmio pelo pó de casca de coco. 94

Figura 36- Representação gráfica para teste de ordem zero . 96

Figura 37 - Representação gráfica para teste de primeira ordem. 97

Figura 38 - Representação gráfica para teste de segunda ordem. 98

Figura 39 - Representação gráfica do modelo depseudo primeira ordem. 99

Figura 40 - Representação gráfica do modelo de pseudo segunda ordem. 100 


\section{Lista de tabelas}

Tabela 1 - Limites máximos aceitáveis de alguns metais em águas segundo EPA e CONAMA.

Tabela 2 - Setores industriais com maior presença de íons de metais pesados nos efluentes. 22

Tabela 3-Classificação de periculosidade dos metais. 22

Tabela 4 - Vantagens e desvantagens dos métodos tradicionais de tratamento de efluentes contendo metais pesados.

Tabela 5 - Classificação de resíduos que podem ser utilizadas como biossorventes.

Tabela 6-Vantagens e desvantagens da utilização de biomassas. 35

Tabela 7-Capacidade de remoção do cádmio por diferentes biomassas. $\quad 39$

Tabela 8- Caracterização química típica da casca de coco verde. 40

Tabela 9- Composição química da casca de coco e da fibra de coco. 41

Tabela 10 - Freqüência no infravermelho das bandas de alguns grupos funcionais presentes no pó de casca de coco verde. 42

Tabela 11 - Comparação entre adsorção física e química. 45

Tabela 12 - Modelos de isotermas de adsorção de Langmuir .e Freundlich. $\quad 46$

Tabela 13 - Condições operacionais do espectrofotômetro. 65

Tabela 14 - Distribuição granulométrica do pó de casca de coco verde. 68

Tabela 15 - Efeito do tamanho de partícula na biossorção. 79

Tabela 16 - Efeito da concentração inicial dos íons na biossorção. 86

Tabela 17 - Constantes de adsorção do Cd, Cr (III) e Cr (VI). 90

Tabela 18 - Comparação da capacidade de remoção entre diferentes biossorventes para o $\mathrm{Cr}(\mathrm{VI}) \quad 92$

Tabela 19 - Comparação da capacidade de remoção entre diferentes biossorventes para o $\mathrm{Cd}$. 93

Tabela 20 - Comparação da capacidade de remoção entre diferentes biossorventes para o $\mathrm{Cr}$ (III) . 93

Tabela 21 - Dados utilizados na determinação da ordem do processo de biossorção,

Tabela 22 - Parâmetros cinéticos segundo o modelo de pseudo primeira ordem e de pseudo segunda ordem. 


\section{Lista de símbolos}

q: $\quad$ Quantidade de adsorvato retido no sólido, no equilíbrio (mg/g).

q max: $\quad$ Parâmetro de Langmuir, relativo à capacidade de adsorção $(\mathrm{mg} / \mathrm{g})$

$\mathrm{K}_{\mathrm{ads}}$ : $\quad$ Constante de Langmuir, relativo a energia de adsorção (L/mg).

C: $\quad$ Concentração na solução quando está em equilíbrio $(\mathrm{mg} / \mathrm{g})$.

Kf: $\quad$ Constante de Freundlich, relativo a capacidade de adsorção (L/g).

$\mathrm{n}$ : $\quad$ Constante de Freundlich, relativa à intensidade de adsorção.

$\xi$ : $\quad$ Potencial zeta $(\mathrm{mV})$.

M: $\quad$ Mobilidade eletroforética ( $\mu \mathrm{cm} / \mathrm{vs})$.

$\eta: \quad$ Viscosidade do fluido (Poise).

$\varepsilon$ : $\quad$ Permisividade da suspensão (F/cm).

t Tempo (minutos).

$r_{\mathrm{A}}: \quad$ Velocidade de reação ou taxa de conversão do reagente $\mathrm{A}$.

k: $\quad$ Constante de velocidade de reação.

$\mathrm{C}_{\mathrm{A}}$ : Concentração do reagente $\mathrm{A}$.

$\mathrm{C}_{\mathrm{AO}}$ : Concentração inicial do reagente $A$.

$\mathrm{X}_{\mathrm{A}}$ : $\quad$ Fração convertida do reagente $\mathrm{A}$.

$\mathrm{q}_{\mathrm{t}}$ - Quantidade de adsorvato retido no sólido no tempo t.

$\mathrm{k}_{1}$ : $\quad$ Constante de velocidade de reação de pseudo-primeira ordem (1/min).

$\mathrm{k}_{2}$ : $\quad$ Constante de velocidade de reação de pseudo-segunda ordem (g/mg.min).

$\mathrm{C}_{0}$ : $\quad$ Concentração inicial dos íons na solução (ppm).

$\omega: \quad$ Velocidade de agitação (rpm).

$\mathrm{C}_{\text {biomassa }}$ : Concentração da biomassa $(\mathrm{g} / \mathrm{L})$.

PS: $\quad$ Tamanho de partícula $(\mathrm{mm})$.

T: $\quad$ Temperatura $\left({ }^{\circ} \mathrm{C}\right)$. 